\title{
Fault Diagnosis Technology of Reciprocating Pumps based on Inlay Model Wavelet Neural Network ZHAO Zhi-hua ${ }^{1}$, WU Li ${ }^{2}$
}

\author{
1. Academy of Electric Information Engineering, Northeast petroleum \\ University Daqing 163318 China;
}

\begin{abstract}
2. Training Center, Natural gas branch of Daqing Oilfield, Daqing 163412 ChinaAbstract: A method is proposed based on inlay model Wavelet Neural Network in order to determine the reciprocating pump fault type accurately. This paper used the reciprocating pump single cylinder pressure signals as the characteristics of the system signal by wavelet packet decomposition to extract fault features vector, which is the input of wavelet neural network, and at the same time, used wavelet neural network to determine the type of the fault. Diagnosis of faults of fluid end on a reciprocating pump proves the system fault diagnosis accuracy to $94 \%$.
\end{abstract}

Key Words: wavelet neural network, Reciprocating Pump, Fault Diagnosis, Wavelet packet

\section{INTRODUCTION}

Reciprocating pump is an important instrument in oil fields, which has been used widely in drilling, oil displacement, and fracturing technology. It is also a complex mechanical system. The key component of its hydralic end is pump value components, but it is damaged easily because of bad working conditions. So it's very significant for state monitoring and fault diagnosis to make reasonable maintenances ${ }^{[1]}$. Domestic and international scholars did a number of researches about reciprocating pump fault diagnosis, and put forward many methods to improve the accuracy continuously. Yet due to complex working conditions, there are so many factors that affect its fault diagnosis. Meanwhile the nonlinear relationship is pretty complicated between reciprocating pump and pump valve components, so the precise mathematical model is difficult to establish which is suitable for all occasions.

Wavelet neural network is an emerging mathematical modeling analysis method in recent years, which combines analysis with neural network theory. Introduce two new parameters - expansion coefficient and translation coefficient, wavelet neural network has more degrees of freedom. The ability of function approximation is more flexible and effective, and fault-tolerant ability is much stronger ${ }^{[2]}$. All of these can overcome some inherent defects of the neural network model. Wavelet packet analysis is also a kind of good time-frequency analysis method, because signals can be localized to be time domain and frequency domain, and it has strong feature extraction function to signals at the same time, which is an effective method of reciprocating fault feature extraction. This article unifiles the feature extraction of wavelet packet analysis and the pattern of adaptive wavelet neural network organically together to diagnosis and prove validity of the proposed algorithm through all the experiments.

\section{Based on Wavelet Packet Fault Feature Extraction}

Compared with wavelet analysis, wavelet packet analysis is a much more detailed decomposition and analyzes further to the part of high frequency in multi-resolution analysis which is not segmented so as to increase the time-frequency analysis rate. That can make fault feature extraction continue smoothly in a more detailed frequency band[3]. When using wavelet packet fault feature extraction, the first is wavelet packet decomposition to original signals. Because the energy of faults is different in different frequency bands. We can get the changing relationship between varies faults and energy according to wavelet packet decomposition coefficient. At the same time, we can establish fault characteristics of energy to complete fault feature extraction.

\subsection{Wavelet Packet Algorithm}

Suppose $^{X_{i j}}$ to be the decomposition coefficient of the original signal S with wavelet packet decomposition. Then reconstruct every decomposition coefficient of wavelet packet and extract the signals in each band rang. If $S_{i j}$ is single reconstruction signal, the total signal $\mathrm{S}$ is:

$$
S=\sum_{j=1}^{2^{i}} S_{i j}
$$

1.2 Fault Feature Extraction 
When something wrong with reciprocating pump, there are huge differences about output signals shown in the same frequency band energy. Thus, we regard the energy of output signal $S_{i j}$ in each frequency as the fault feature vector, which is the fault features of reciprocating pump.

Because original and structure signals are both random, we can write:

$$
E_{i j}=\int\left|S_{i j}(t)\right|^{2} d t=\sum_{k=1}^{n}\left|x_{j k}\right|^{2}
$$

(where ${ }^{x_{j k}}(k=1,2, \cdots, n)$ is the amplitude of discrete point of construction signal)

Structure feature vector as follows:

$$
T=\left[E_{i 1}, E_{i 2}, E_{i 3}, \cdots, E_{i 2}{ }_{i{ }^{i}}\right]
$$

To avoid inconveniences when energy is growing bigger, we normalized $\mathrm{T}$ :

$$
T^{\prime}=\left[E_{i 1} / E, E_{i 2} / E, E_{i 3} / E, \cdots, E_{i 2} / E\right]
$$

The normalized eigenvectors are input of Wavelet neural network.

2 For the Valve Fault Diagnosis Based on Wavelet Neural Network

\subsection{Adaptive Wavelet Neural Network}

This article adapts inlay model wavelet neural net, which fuses the wavelet decomposition and feed forward neural network directly that is using wavelet function instead of hidden nodes of neural network function .At the same time , the weights of corresponding input layer to hidden layer is instead by scale factor of wavelet function, and threshold of output layer to hidden layer is also instead by translation factor of wavelet function. In adaptive wavelet neural network, because of the low correlation of wavelet neuron, it makes its convergence speed faster, approximation and fault tolerant ability stronger[4,5].The inlay model wavelet neural network is expressed in Figure 1.

The number of nodes in input is $\mathrm{M}$, where $x_{i}(i=1,2, \cdots, M)$ are input samples; The number of nodes in hidden layer is $\mathrm{N}$, where $h_{j}(j=1,2, \cdots, K)$ is the wavelet function. The ${ }^{j}$ wavelet basis function of neuron in hidden

$$
\text { layer is } h\left(\frac{x-b_{j}}{a_{j}}\right) \text {.Meanwhile, there are } \mathrm{K} \text { nodes in output layer: } y_{k}(k=1,2, \cdots, N) .^{\omega^{j i} \text { means }}
$$

connection weights of the neuron in input layer to the $j$ in hidden layer; ${ }^{\omega}{ }_{j k}$ means connection weights of the $j$ neuron in input layer to the $k$ in output layer; $a_{j}, b_{j}$ are scale coefficient and translation factor namely. The output of wavelet neural network could be:

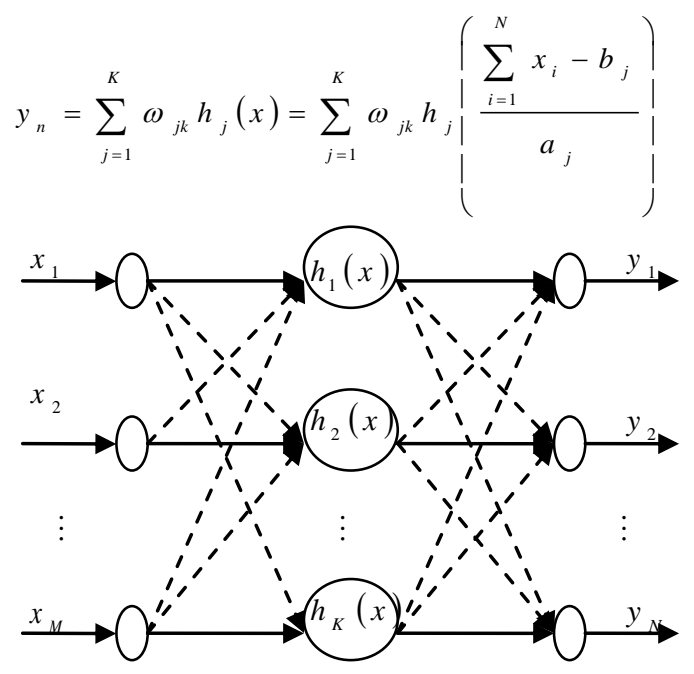

Fig.1 Inlay model wavelet neural network

2.2 Wavelet Neural Network Training Algorithm

] 


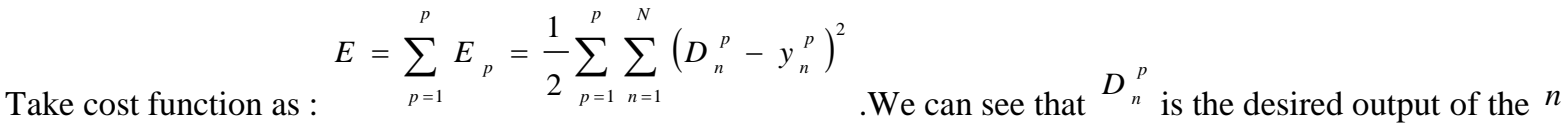
node in output layer; $y_{n}^{p}$ is the actual output on net; ${ }^{E}{ }_{p}$ is the error of the ${ }^{p}$ sample.Actually, the adjustment essence of wavelet neural network weights is gradient descent method[6,7] . Its inherent features make the training process of wavelet neural network exist some disadvantages as follows: slow convergence speed, easy to be trapped in local minima, easy to cause shock effects, etc.

In view of above shortcomings, there are two improvements : one is introducing momentum item, the other is adopting the way of variable learning rates. Gradient descent back propagation algorithm is used which combines adaptive learning rate with momentum in this paper.

(1)If $E(k)>\xi * E(k-1),(1 \%<\xi<5 \%)$, that is the learning error this time is more than last, we will cancel it. $\eta(k)=\eta(k-1) \times l, \& 0<l<1$; so momentum coefficient $\alpha=0$.

(2) If $E(k)<E(k-1)$, that is the learning error this time is less than last, we will keep it. $\eta(k)=\eta(k-1) \times g, \&^{g>1}$; if momentum coefficient is set 0 , it will be back to the initial value.

(3)If $E(k-1)<E(k)<\xi * E(k-1)$, keep this learning and learning rate $\eta(k)$ unchanged . If momentum coefficient was set 0 , it will be back to the initial value.

The threshold in neural network and expansion factor and translation factor could be adjusted as below:

$$
\begin{gathered}
\omega_{j k}(k+1)=\omega_{j k}(k)-\eta_{\omega} \frac{\partial E}{\partial \omega_{j k}}+\alpha_{\omega} \Delta \omega_{j k}(k) \\
a_{j}(k+1)=a_{j}(k)-\eta_{a} \frac{\partial E}{\partial a_{j}}+\alpha_{a} \Delta a_{j}(k) \\
b_{j}(k+1)=b_{j}(k)-\eta_{b} \frac{\partial E}{\partial b_{j}}+\alpha_{b} \Delta b_{j}(k)
\end{gathered}
$$

Where: $\eta_{\omega}, \eta_{a}, \eta_{b}$-learning rates of parameters $\omega_{j k}, a_{j}, b_{j} ; \alpha_{\omega}, \alpha_{a}, \alpha_{b}$-movement factor of parameters

$$
\omega_{j k}, a_{j}, b_{j} .
$$

Its training steps are as follows:

(1) initialize network and parameters: Assign an initial value to expansion coefficient ${ }^{a}$, translation factor ${ }^{b}$, network weight coefficient ${ }^{\omega_{i j}},{ }^{\omega_{j k}}$, learning rate ${ }^{\eta}$, momentum coefficient $\alpha$;

(2)Input learning sample ${ }^{X_{n}}$, and calculate actual output and learning errors;

(3)Error Back Propagation, and correct each parameter;

(4) judge errors, according to Gradient descent back propagation algorithm ,introduced in the article to update learning rates and momentum factors;

(5) calculate whether the error value ,trained, can meet the demands .If so, finish the training. If not, go back to step two until the global error function is less than the preset minimum value [8].

3 Reciprocating Pump Fault Diagnosis

For reciprocating pump fault diagnosis, we can use pressure signals in single pump cylinder as system characteristic signal to extract fault feature vectors. In this paper, the collected original pressure signals are decomposed 3 layers wavelet packet and composed by 8 eigenvector to extract fault features of reciprocating pump and as the input vector of the wavelet neural network[7]. So the input nodes are 8. The author of the paper diagnosis respectively about the faults of reciprocating pump seals,pump wear and spring brakeage, (001), (010)and (100) representing the three working conditions respectively. Because they are the target output of the net so the output nodes are 3.Wavelet neural network adopt 3 layer structure, hidden layer wavelet is Morlet- wavelet. We use the way of step test to determine the number of hidden layer nodes.

Firstly, suppose we consider an error $E^{*}$, and get a smaller hidden layer node to calculate its another error $E$. Secondly, compare $E$ and $E^{*}$.If $E<E^{*}$, it is the hidden layer node which we need. Or, add 1 to the node and 
continue to calculate until it is $E<E^{*}$, that is to say, we can ensure the hidden layer nodes. After the experiments, the hidden layer node is 12 . We make the learning rate of initial value $\eta=0.5$, momentum factor $\alpha=0.85$, and the training error accuracy requirement is 0.005 . We use 12 bad seal samples, 12 valve abrasion samples and 12 spring fracture samples to train the net with the adaptive wavelet neural network designed in the paper. Through the learning of 358 steps, the net has achieved the accuracy requirement.

The result of it is shown in Fig. 2 :(a)is learning rate change curve (b)is training errors curve 12 groups training

sample corresponding network actual output as is shown in Tab.1.

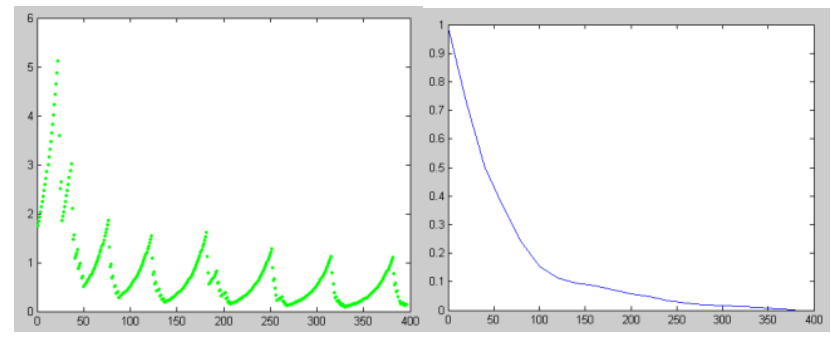

(a) Learning rate curve (b) Error curve

Fig. 2 The result of the training

Tab.1 Output value of after training the network output node

\begin{tabular}{|c|c|c|c|}
\hline state & \multicolumn{3}{|c|}{ Diagnosis output } \\
\hline Seal bad & 0.0000 & 0.0002 & 0.9878 \\
Pump valve & 0.0065 & 1.0004 & 0.0081 \\
damaged & 0.9985 & 0.0099 & 0.0202 \\
Spring damaged & 0.0010 & 0.0389 & 1.0728 \\
Seal bad & 0.0000 & 0.9256 & 0.0001 \\
Pump valve & 0.9601 & 0.0096 & 0.0586 \\
damaged & 0.0402 & 0.0401 & 0.9800 \\
Spring damaged & 0.0498 & 0.9899 & 0.0689 \\
Seal bad & 0.9049 & 0.0027 & 0.0054 \\
Pump valve & 0.0029 & 0.0068 & 1.0257 \\
damaged & 0.0103 & 0.9827 & 0.0254 \\
Spring damaged & 0.9576 & 0.0521 & 0.0026 \\
Seal bad & & & \\
Pump valve & & & \\
damaged & & & \\
Spring damaged & & & \\
\hline \multicolumn{4}{|l}{}
\end{tabular}

Knowing now that the net after rained can express the given knowledge pretty well. Input the samples which is diagnosed with that kind of network, we can get other samples and diagnostic results in Tab.2. 
Tab.2 The test sample and results

\begin{tabular}{c|c|c|c}
\hline \hline & \multicolumn{3}{|c}{ Fault types } \\
\cline { 2 - 4 } & Seal bad & $\begin{array}{c}\text { Pump valve } \\
\text { damaged }\end{array}$ & $\begin{array}{c}\text { Spring } \\
\text { damaged }\end{array}$ \\
\hline \multirow{4}{*}{ Validation } & 0.0000 & 0.0002 & 0.0078 \\
data & 0.9965 & 10.004 & 5.6581 \\
& 1.5985 & 1.6999 & 0.8202 \\
& 0.0000 & 1.0389 & 1.0728 \\
& 0.2601 & 0.0000 & 0.0001 \\
& 0.6402 & 0.04096 & 0.5986 \\
Diagnosis & 0.2498 & 0.2899 & 0.0800 \\
output & 0.0012 & 0.0075 & 0.0689 \\
& 0.0027 & 1.0036 & 0.9875 \\
& 0.9965 & 0.0125 & 0.0021 \\
& Fault 1 & Fault 2 & 0.0289 \\
& $(001)$ & $(010)$ & Fault 3 (100) \\
\hline \hline
\end{tabular}

According to the European norm theory, the test errors of the data are 0.0035、0.002 and 0.0043, which are very small. The most important is that the data accuracy of reciprocating pump fault diagnosis could increase to $94 \%$.

\section{CONCLUSION}

This paper mainly studies the changing signals in non-stationary reciprocating pump with wavelet packet analysis technology. And decompose them into different levels and frequency bands, extract different feature vector of faults of reciprocating pumps, which can reflect effectively; and at the same time, send it to wavelet neural network which has the function of state recognition. We also adopt the gradient descent back propagation algorithm combined adaptive learning rate with momentum. Not only can it improve convergence speed, but also avoid local minimum. After experimental verification, the data accuracy of fault diagnosis algorithm could increase to $94 \%$. So the paper has certain application value.

\section{REFERENCES}

[1] Duan Yubo, You Dawei. A Survey of Monitoring and Intelligent Fault Diagnosis for the Pump Valves of Reciprocating Pump[J].Techniques of automation and Applications,2003,22(9):1-4.

[2] Wang Naimin, Liu Runhua, Xiao Guangyun. Application research of wavelet neural network in Crude oil moisture content prediction [J]. Chemical instrument and automation, 2008,35(3):51-53.

[3] Xu Jianjun, Yang Shiyan. Fault diagnosis of wavelet packet neural network on pump valves of reciprocating pump based on pressure signal[J]. Journal of Dalian Maritime University, 2007,33(3):22-25.

[4] Ma Rui, The principle of artificial neural network[M]. Peking: China Machine Press,2010,7.

[5] Zhang Qinghua and Benveniste A Wavelet networks. IEEE trans on NN[J], 1992, 3(6): 889-898.

[6] Liu Fen ,Pan Hongxia. Study on Application of Optimizing Wavelet Neural Network for Gear Box Fault Diagnosis [J]. Noise and vibration control. 2011, 10(5):146-149.

[7] Zhong Jingchen. Reserch Of Fault Diagnosis Method Based on Wavelet Neural Network[D] Masterdegree thesis. Qingdao: Chinese Marine University, 2004.

[8] Wang Xin, Dai Li. Application of Improved Wavelet Neural Network in Fault Diagnosis Infrequency Conversion System[J]. Noise and vibration control. 2008, 12(6):20-23. 